\title{
TATA INSTITUTE RADIOCARBON DATE LIST XI
}

\author{
D P AGRAWAL and SHEELA KUSUMGAR
}

Tata Institute of Fundamental Research, Bombay-400 005, India

This is the last installment of ${ }^{14} \mathrm{C}$ dates done at the Tata Institute; the lab has now shifted to the Physical Research Laboratory, Navarangpura, Ahmedabad-380 009, India.

The value $\tau 1 / 2=5568$ yr has been used to calculate all $\mathrm{BP}$ dates. Dates were converted to $\mathrm{AD} / \mathrm{BC}$ scale by using 1950 as the reference year. The NBS oxalic acid was used as the modern standard.

We have measured the ${ }^{14} \mathrm{C}$ activity of the methane samples in gas proportional counters. The samples were converted to methane by using a reactor described earlier (R, 1971, v 13, p 442-449).

This date list includes dates on some old mining areas, some important Stone Age dates, and some measurements of various Quaternary processes including eustatic studies on the $\mathrm{W}$ coast of India. The hydrospheric samples include some dates done to study groundwater recharge problems in W India. The Pacific Ocean samples were measured to study the siltation and dissolution rates of calcareous particles in transit through a sea-water column. A series of Egyptian well-dated historic samples were measured to study the ${ }^{14} \mathrm{C} /{ }^{12} \mathrm{C}$ variations in the past.

General Comment*: for the first time, an Upper Palaeolithic level has been dated to ca 20,000 вс (TF-1245) from U P. The microlithic occupation at Sarai Nahar Rai was dated ca 1000 BC (TF-1356, -1359) based on charred bones. Prehistoric deposits from a Ceylonese cave was dated to са 6000 вс (TF-1074). A Painted Grey Ware, Iron age deposit from U P is dated ca $500 \mathrm{BC}$ (TF-1228).

\section{ACKNOWLEDGMENT}

We are thankful to $\mathrm{R} \mathrm{K}$ Pant for help in preparing the manuscript.

\section{SAMPLE DESCRIPTIONS}

\section{ANCIENT MINING SAMPLES}

\section{TF-1199. Kolar, India, old gold works}

$1260 \pm 85$

Charcoal from excavations of an old mine $\left(12^{\circ} 57^{\prime} \mathrm{N}, 78^{\circ} 16^{\prime} \mathrm{E}\right)$, Dist Kolar. Subm by T G Varghese, Bhabha Atom Res Centre, Bombay.

\section{Kumbaria old mining series, Gujarat}

Kumbaria $\left(24^{\circ} 19^{\prime} \mathrm{N}, 72^{\circ} 51^{\prime} \mathrm{E}\right)$, Dist Banaskantha. Subm by N C Shekhar, Min Expl Corp, Banaskantha. Samples assoc with slag of old smelting of copper, lead, silver, etc, lying on surface.

\section{TF-1221. Charcoal}

\section{Charcoal extracted from slags.}

* For these comments, dates are based on $\tau 1 / 2=5730 \mathrm{yr}$. 


\section{TF-1222. Charcoal}

Charcoal extracted from slags.

II. ARCHAEOLOGIC SAMPLES

TF-1245. R Belan, India, Gravel III

$19,160 \pm 330$

$17,210 \mathrm{BC}$

Shells from Gravel III on R Belan $\left(24^{\circ} 54^{\prime} \mathrm{N}, 82^{\circ} 2^{\prime} \mathrm{E}\right)$, Dist Allahabad. Subm by Dir Inst Archael, Allahabad. Comment: an Upper Palaeolithic industry is assoc with Gravel III.

\section{$7640 \pm 110$ \\ TF-1094. Beli Lena Athula, Ceylon, cave remains \\ 5690 BC}

Carbonized kernels at $.45 \mathrm{~m}$ depth, from a prehistoric cave deposit $\left(6^{\circ} 56^{\prime} 5^{\prime \prime} \mathrm{N}, 80^{\circ} 14^{\prime} 5^{\prime \prime} \mathrm{E}\right)$, near Maniyangama. Subm by Vishnu Mittre, Birbal Sahni Inst Palaeobot, Lucknow.

TF-1162. Gharluli, Afghanistan, Late Neolithic

Modern

Charcoal from Gharluli $\left(35^{\circ} 45^{\prime} \mathrm{N}, 65^{\circ} 00^{\prime} \mathrm{E}\right)$, Dist Maimana, Trench 1, Cut 2de, 6m, Sample 16/2d-600/8-9-69. Subm by L Dupree, Pennsylvania State Univ, Philadelphia. Comment (L D): nomads dug pits at site up to modern times, disturbing underlying deposits.

TF-1330. Inamgaon, India, Chalcolithic

$3090 \pm 100$

Wood charcoal from Inamgaon $\left(18^{\circ} 35^{\prime} \mathrm{N}, 74^{\circ} 32^{\prime} \mathrm{E}\right)$, Dist Poona, a Chalcolithic site, Loc E7, Layer 4. Subm by Dir, Deccan College, Poona.

TF-1228. Khalaua, India, P G Ware level

$$
\begin{gathered}
2420 \pm 95 \\
470 \mathrm{BC}
\end{gathered}
$$

Charcoal from Khalaua $\left(27^{\circ} 6^{\prime} \mathrm{N}, 77^{\circ} 52^{\prime} \mathrm{E}\right)$, Dist Agra, Loc Khl-L, II-IV(a), Layer 9, depth $2.6 \mathrm{~m}$ to $2.75 \mathrm{~m}$. Subm by Dir Gen, Archaeol, New Delhi.

TF-1356,

TF-1359. Sarai Nahar Rai, India Microlithic(?)

$2860 \pm 120$

Charred and semi-charred bones from Sarai Nahar Rai $\left(25^{\circ} 48^{\prime} \mathrm{N}\right.$, $\left.81^{\circ} 50^{\prime} \mathrm{E}\right)$, Dist Pratapgarh, a Mesolithic site, Hearth 1/A3 and 2/B4, depths 2 to $4 \mathrm{~cm}$ to 5 to $6 \mathrm{~cm}$. Subm by Dir, Inst Archaeol, Allahabad. Comment: date younger than uncharred bones dated earlier (TF-1104: $10,050 \pm 110)$.

TF-1301. Surkotada, India, Harappa culture

$3840 \pm 130$ $1890 \mathrm{BC}$

Charcoal from Surkotada $\left(23^{\circ} 37^{\prime} \mathrm{N}, 70^{\circ} 50^{\prime} \mathrm{E}\right)$, Dist Kutch, a fortified Harappan site, Loc B1, Qd 3, Layer 17, depth $5.65 \mathrm{~m}$. Subm by Dir Gen Archaeol, New Delhi. 


\section{EGYPTIAN HISTORIC SAMPLES}

General Comment: these samples were measured to determine ${ }^{14} \mathrm{C} /{ }^{12} \mathrm{C}$ variations in the past. Though $\delta^{13} \mathrm{C}$ values are given, dates are not corrected for this effect.

\section{Egyptian Series I}

Samples subm by W F Libby.

TF-562. Sneferu

$$
\begin{gathered}
4310 \pm 105 \\
2360 \mathrm{BC} \\
\delta^{1 s} C=-21.28 \%
\end{gathered}
$$

Wood from tomb of Sneferu at Meydum. Comment: sample same as C-12 (Libby, 1965).

\section{TF-563. Hemaka}

$$
\begin{aligned}
& 4580 \pm 60 \\
& 2630 \mathrm{BC} \\
& \delta^{13} C=-25.63 \%
\end{aligned}
$$

Wood from tomb of Vizir Hemaka, contemporary of King Udimu, First Dynasty, at Sakkara. Average of 3 measurements: 4510, 4575, and 4610 yr. Comment: sample same as C-267.

\section{TF-564. Sesostris III}

$$
\begin{gathered}
3570 \pm 75 \\
1620 \text { BC } \\
\delta^{1 s} C=-19.40 \%
\end{gathered}
$$

Wood from funerary ship from tomb of Sesostris III. Average of 2 measurements: 3560 and 3570 yr. Comment: sample same as C-81.

TF-567. Zoser

$4180 \pm 80$ 2230 BC $\delta^{13} \mathrm{C}=-24.54 \%$ o

Piece of Acacia wood from Zoser's Step Pyramid at Sakkara. Average of 2 measurements: 4135 and 4205 yr. Comment: sample same as C-1.

TF-568. Zoser

$4130 \pm 50$

2180 BC

$\delta^{13} \mathrm{C}=-26.41 \%$ 。

Piece of Sycamore wood from Zoser Step Pyramid at Sakkara. Average of 4 measurements: $4305,4220,4090$, and $3830 \mathrm{yr}$.

\section{Egyptian Series II} UAR.

Well-dated historic samples from Egypt. Subm by Chairman, AEC,

\section{TF-1208. Reeds}

$3840 \pm 135$

$1890 \mathrm{BC}$

Reeds from tomb of Ones Re, No. 463, Old Kingdom, Luxor. Comment: archaeologic date ca $2100 \mathrm{BC}$.

TF-1209. Reeds

$3010 \pm 80$

$1060 \mathrm{BC}$

Reeds from wall of store room of temple Ramseum, Rameses II. Comment: archaeologic date ca $1250 \mathrm{BC}$. 


\section{TF-1211. Cloth}

Cloth, 22nd Dynasty, Luxor.

TF-1212. Wood

Door of tomb Mono Mhat, No. 34, Assasee of 26th Dynasty. Comment: archaeologic date ca 700 вс.

\section{QUATERNARY SAMPLES}

\section{Quaternary sediment series, W Rajasthan}

Samples subm by R P Dhir, Cent Arid Zone Res Inst, Jodhpur.

General Comment: samples measured to study onset of dessication in W Rajasthan.

\section{TF-1214. Concretionary deposit}

$$
\underbrace{+1985}_{27,880}
$$

Calcium carbonate from $15 \mathrm{~km}$ of Pokran, concretionary layer at 38 to $100 \mathrm{~cm}$ below aeolian sand.

\section{TF-1215. Concretionary deposit}

$14,080 \pm 170$

Calcium carbonate, Dodo-hill, piedmont slope, concretionary layer over rhyolite zone of weathering.

\section{TF-1089. Panambur Harbour Area, India,} coastal sediments

$$
\begin{array}{r}
37,380 \\
35,430 \text { BC }
\end{array}
$$

Carbonized wood from tree root, depth $12 \mathrm{~m}$, ancient coastal sediment $\left(12^{\circ} 56^{\prime} \mathrm{N}, 74^{\circ} 50^{\prime} \mathrm{E}\right)$, Dist S Kanara. Subm by E V Nielson, Port Trust, Cochin. Comment: sample dated to study coastal siltation rates.

\section{Coastal sediments series, Maharashtra}

Samples subm by D P Agrawal and S Guzder, TIFR, Bombay.

General Comment: samples measured to study Quaternary eustatic changes on W coast, India (Agrawal et al, 1972). Wherever depths have been given below surface, there still is uncertainty about their exact relationship with HWL.

TF-555. Kolthara-Dabhol, coastal sediments

$1520 \pm 90$

Shells from Kolthara-Dabhol $\left(17^{\circ} 39^{\prime} 10^{\prime \prime} \mathrm{N}, 73^{\circ} 10^{\prime} 50^{\prime \prime} \mathrm{E}\right)$, Dist Ratnagiri, depth $-1.8 \mathrm{~m}, 105 \mathrm{~m}$ inland from sea.

TF-556. Kolthara-Dabhol, coastal sediments $2500 \pm 85$ Shells, depth $-3.8 \mathrm{~m}$. 
TF-557. Kolthara-Dabhol, coastal sediments

$1930 \pm 100$

Shells, depth $-4.25 \mathrm{~m}$.

AD 20

TF-558. Harnai, coastal sediments

$2370 \pm 80$

420 BC

Shells from Harnai $\left(17^{\circ} 49^{\prime} 10^{\prime \prime} \mathrm{N}, 73^{\circ} 8^{\prime} 0^{\prime \prime} \mathrm{E}\right)$, Dist Ratnagiri, $0.5 \mathrm{~m}$ above HWL.

TF-560. Harnai, coastal sediments

$1860 \pm 90$

Shells, $1.7 \mathrm{~m}$ above HWL.

AD 90

TF-1365. Damle Wadi $2710 \pm 105$

uhagar, coastal sediments $760 \mathrm{BC}$

Shells from Damle Wadi Guhager (17 $\left.29^{\prime} 55^{\prime \prime} \mathrm{N}, 73^{\circ} 13^{\prime} 35^{\prime \prime} \mathrm{E}\right)$, Dist Ratnagiri, depth $-1.1 \mathrm{~m}, 50 \mathrm{~m}$ inland from beach.

TF-1366. Damle Wadi Guhagar, coastal sediments $\begin{gathered}2160 \pm 90 \\ 210 \mathrm{BC}\end{gathered}$

Shells, depth $-2.20 \mathrm{~m}$.

$\begin{array}{ll}2070 & \pm 125\end{array}$

TF-1367. Damle Wadi Guhagar, coastal sediments 120 BC

Shells, $4 \mathrm{~m}$ below surface.

$3890 \pm 110$

TF-1368. Khare Wadi Guhagar, coastal sediments $\begin{aligned} & 3890 \pm \\ & 1940 \mathrm{BC}\end{aligned}$

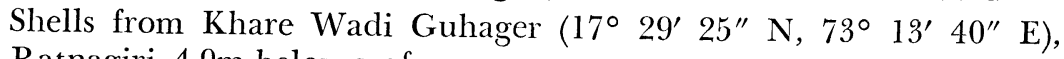
Dist Ratnagiri, $4.9 \mathrm{~m}$ below surface.

TF-1371. Devgad, coastal sediments

$1950 \pm 100$

Shells from Devgad (16 $22^{\prime} 30^{\prime \prime} \mathrm{N}, 73^{\circ} 24^{\prime} 50^{\prime \prime}$ E), Dist Ratnagiri, 3 to $4 \mathrm{~m}$ above HWL.

TF-1372. Malvan, coastal sediments

$$
1080 \pm 105
$$
Dist Ratnagiri, $1.4 \mathrm{~m}$ above HWL.

TF-1374. Malvan-Vaiyri, coastal sediments

$2190 \pm 145$

240 BC

Shells from Malvan-Vaiyri $\left(16^{\circ} 1^{\prime} 35^{\prime \prime} \mathrm{N}, 73^{\circ} 31^{\prime} 50^{\prime \prime} \mathrm{E}\right)$, Dist Ratnagiri, $9 \mathrm{~m}$ below surface.

Coastal sediments series, Australia

Samples subm by E D Gill, Nat Mus Victoria, Melbourne.

TF-1381. S Coast of New South Wales, coastal $150 \pm 80$ sediments

AD 800

Aragonitic shells from shell grit zone of headland between Norrawallee beach and Norrawalle inlet, off Ulladulla, ca $2 \mathrm{~m}$ above MSL, covered with soil, No. 11/1772. 


\section{TF-1382. SW of Boggaley Creek, coastal} sediments

$340 \pm 85$

AD 610

Mollusk shells from cemented calcarianite beach rock overlying a pebble bed, at SW end of a small prograded embayment SW of Boggaby Creek, Victoria, No. 12/1972.

\section{HYDROSPHERIC SAMPLES}

\section{Gujarat groundwater series}

Samples subm by B S Sukhija, TIFR, Bombay, to study recharge of aquifers in region.

\begin{tabular}{|c|c|c|c|c|c|}
\hline $\begin{array}{l}\text { Sample } \\
\text { no. }\end{array}$ & Location & Well type & Depth & $\begin{array}{c}\delta^{14} \mathrm{C} \\
\% \text { modern }\end{array}$ & $\begin{array}{l}\text { Aquifer } \\
\text { no. }\end{array}$ \\
\hline TF-1184 & $\begin{array}{l}\text { Maktapur, } \\
\text { Dist Mehsana } \\
\left(23^{\circ} 42^{\prime} \mathrm{N}\right. \\
\left.72^{\circ} 30^{\prime} \mathrm{E}\right)\end{array}$ & Tube-well & $\begin{array}{l}320 \mathrm{~m} \\
\text { to } \\
326 \mathrm{~m}\end{array}$ & $37.4 \pm 0.9$ & $\begin{array}{l}\text { Single } \\
\text { aquifer } \\
\text { tapped }\end{array}$ \\
\hline TF-1185 & $\begin{array}{l}\text { Sipor, } \\
\text { Dist Mehsana, } \\
\left(23^{\circ} 40^{\prime} \mathrm{N},\right. \\
\left.72^{\circ} 50^{\prime} \mathrm{E}\right)\end{array}$ & -do- & $65 \mathrm{~m}$ & $72.8 \pm 0.9$ & $\begin{array}{l}\text { Recharge } \\
\text { area }\end{array}$ \\
\hline
\end{tabular}

\section{Rajasthan groundwater series}

Samples subm by V N Nijampurkar, TIFR, Bombay, to study aquifer recharge in area.

\begin{tabular}{|c|c|c|c|c|c|}
\hline $\begin{array}{l}\text { Sample } \\
\text { no. }\end{array}$ & Location & Well type & Depth & $\begin{array}{c}\delta^{14} \mathrm{C} \\
\% \text { modern }\end{array}$ & $\begin{array}{l}\text { Aquifer } \\
\text { no. }\end{array}$ \\
\hline TF-1122 & $\begin{array}{l}\text { Ajasar, Dist } \\
\text { Jaisalmer } \\
\left(27^{\circ} 15^{\prime} \mathrm{N},\right. \\
\left.71^{\circ} 43^{\prime} \mathrm{E}\right)\end{array}$ & Tube-well & $\begin{array}{l}100 \mathrm{~m} \\
\text { to } \\
117 \mathrm{~m}\end{array}$ & $33.8 \pm 0.8$ & Second \\
\hline TF-1151 & $\begin{array}{l}\text { Chandan, Dist } \\
\text { Jaisalmer } \\
\left(26^{\circ} 59^{\prime} \mathrm{N},\right. \\
\left.71^{\circ} 18^{\prime} \mathrm{E}\right)\end{array}$ & Tube-well & $285 \mathrm{~m}$ & $56.6 \pm 0.7$ & Mixed \\
\hline TF-1154 & $\begin{array}{l}\text { Neron, Dist } \\
\text { Jaisalmer } \\
\left(26^{\circ} 48^{\prime} \mathrm{N},\right. \\
\left.71^{\circ} 28^{\prime} \mathrm{E}\right)\end{array}$ & Dug-well & $38 \mathrm{~m}$ & $85.3 \pm 1.2$ & Mixed \\
\hline TF-1155 & $\begin{array}{l}\text { Undu, Barmer } \\
\left(26^{\circ} 18^{\prime} \mathrm{N}\right. \\
\left.71^{\circ} 40^{\prime} \mathrm{E}\right)\end{array}$ & Tube-well & $118 \mathrm{~m}$ & $54.8 \pm 1.5$ & \\
\hline
\end{tabular}




\section{Pacific Ocean series}

Subm by B L K Somayajulu, TIFR, Bombay.

General Comment: calcareous material trapped in spongin matrix from Pacific waters at depths 2300 to $3500 \mathrm{~m}$. The ratio ${ }^{14} \mathrm{C} /{ }^{12} \mathrm{C}$ corresponds to values observed in surface water in recent years resulting from additional man-made ${ }^{14} \mathrm{C}$, thus indicating that calcareous particles resulted from recent biologic productivity. Results are related to mean settling rates and sizes and dissolution rates of biogenic calcareous particles in transit through a seawater column.

\begin{tabular}{|c|c|c|c|c|c|c|c|}
\hline $\begin{array}{c}\text { Sample } \\
\text { no. }\end{array}$ & Location & Date & $\begin{array}{c}\text { Depth at } \\
\text { which water } \\
\text { was flushed } \\
\text { (m) }\end{array}$ & $\begin{array}{l}\text { Weight } \\
\text { of } \\
\text { sponges } \\
(\mathrm{kg})\end{array}$ & $\begin{array}{c}\text { Volume } \\
\text { of } \mathrm{CO}_{2} \\
\text { (L) }\end{array}$ & $\begin{array}{c}\delta^{14} \mathrm{C} \\
\% o\end{array}$ & $\begin{array}{c}\Delta^{14} \mathrm{C} \\
\% o\end{array}$ \\
\hline TF-812 & $\begin{array}{l}\text { Nova III } \\
(\text { Sta } 7) \\
\left(16^{\circ} 00^{\prime} \mathrm{N},\right. \\
\left.179^{\circ} 05.7^{\prime} \mathrm{W}\right)\end{array}$ & $6 / 22 / 67$ & $2200-2300$ & 4 & 1.20 & $149 \pm 13$ & $92 \pm 12$ \\
\hline TF-865 & $\begin{array}{l}\text { Nova VI } \\
(\text { Sta 1) } \\
\left(31^{\circ} 41^{\prime} \mathrm{S},\right. \\
\left.177^{\circ} 16.2^{\prime} \mathrm{W}\right)\end{array}$ & $9 / 21 / 67$ & $3400-3500$ & 5 & 3.00 & $57 \pm 13$ & $4.2 \pm 12$ \\
\hline
\end{tabular}

\section{Coral X-radiography series}

Coral was analyzed to determine growth rates of several coral species. Comparison of growth rates with X-radiographs of same samples lends added evidence that bands observed are seasonal and may therefore be used as growth rate indicators. Subm by $S$ Krishnaswamy, TIFR, Bombay.

\begin{tabular}{|c|c|c|c|}
\hline Sample no. & Locality & $\begin{array}{l}\text { Depth in vertical } \\
\text { slice of coral }\end{array}$ & $\delta^{14} \mathrm{C} \%$ modern \\
\hline TF-1317 & Jamnagar & $\mathrm{Gl}, 0-1 \mathrm{~cm}$ & $121.3 \pm 2.0$ \\
\hline TF-1318 & $"$ & $" \quad 1-2 \mathrm{~cm}$ & $124.0 \pm 1.9$ \\
\hline TF-1321 & $"$ & $" \quad 2-3 \mathrm{~cm}$ & $119.0 \pm 1.4$ \\
\hline TF-1322 & $"$ & $\Rightarrow \quad 3-4 \mathrm{~cm}$ & $111.6 \pm 1.7$ \\
\hline TF-1323 & $"$ & $" 4-5 \mathrm{~cm}$ & $107.6 \pm 1.6$ \\
\hline TF-1324 & $"$ & $" 5-6 \mathrm{~cm}$ & $107.2 \pm 1.6$ \\
\hline TF-1325 & $"$ & $" 6-7 \mathrm{~cm}$ & $100.4 \pm 1.7$ \\
\hline TF-1326 & $"$ & $" 7-9 \mathrm{~cm}$ & $100.9 \pm 1.6$ \\
\hline TF-1334 & Sikai & $\mathrm{G} 2,0-1 \mathrm{~cm}$ & $121.9 \pm 1.5$ \\
\hline TF-1335 & $"$ & $" \quad 1-2 \mathrm{~cm}$ & $122.8 \pm 1.4$ \\
\hline TF-1336 & $"$ & $" \quad 2-3 \mathrm{~cm}$ & $121.6 \pm 1.5$ \\
\hline
\end{tabular}

REFERENCES

Agrawal, D P, Avasia, R K, and Guzder, Statira, 1972, A multidisciplinary approach to the Quaternary problems in Maharashtra, in: Agrawal, D $\mathrm{P}$ and Ghosh, A (eds), 1973, Radiocarbon and Indian Archaeology, Bombay, TIFR, p 3-17.

Agrawal, D P, Gupta, S K, and Kusumgar, Sheela, 1971, Tata Institute radiocarbon date list IX: Radiocarbon, v 13, p 442-449.

Libby, W F, 1965, Radiocarbon dating, Chicago, Phoenix Books, p 77-78. 